\title{
A NOTE ON SEGAL ALGEBRAS ON EUCLIDEAN SPACES
}

\author{
HWAI-CHIUAN WANG ${ }^{1}$
}

\begin{abstract}
In this note, we construct and study Segal algebras from three important operators on Euclidean spaces - the Hardy-Littlewood maximal function, the Hilbert transform, and the Sobolov operator.
\end{abstract}

0. Introduction. Throughout this article, we denote by $G$ a locally compact abelian group with dual group $\Gamma, T$ the circle group, and $E_{n}$ the $n$-dimensional Euclidean space.

For the convenience of the readers, we recall some definitions:

0.1 Definition. A linear space $S$ of $L^{1}(G)$ is called a homogeneous Banach space if $S$ is a Banach space under some norm \|\|$_{S} \geqslant\|\|_{L^{1}}$, which has the properties:

(H-1) If $f \in S$ and $x \in G$, then

$$
f_{x}(y)=f(y-x) \in S \text { and }\left\|f_{x}\right\|_{S}=\|f\|_{S} .
$$

(H-2) The map $x \rightarrow f_{x}$ is a continuous map of $G$ into $S$.

With the aid of vector-valued integrals, it may be shown that $S$ is a Banach algebra in $L^{1}(G)$, which we call a homogeneous Banach algebra. If $S$ is dense in $L^{1}(G)$, it is called a Segal algebra. For fundamental results on these algebras, see Reiter [3], [4], and Wang [7].

0.2. Definition. A homogeneous Banach algebra $S$ on $G$ is character invariant if $\gamma f \in S$ for all $f \in S, \gamma \in \Gamma$, where $\gamma f(x)=(x, \gamma) f(x)$ for all $x \in G$.

0.3. Definition. A homogeneous Banach algebra $S$ has weak factorization if for every $f \in S$, there are $g_{1}, \ldots, g_{n}, h_{1}, \ldots, h_{n} \in S$ such that $f=\sum_{i=1}^{n}$ $g_{i} * h_{i}$.

1. Hardy-Littlewood maximal function. Given a function $f \in L^{p}\left(E_{n}\right), 1 \leqslant p$ $\leqslant \infty$, the Hardy-Littlewood maximal function $m_{f}$ of $f$ is defined at $x$ by

$$
m_{f}(x)=\sup _{r>0} \frac{1}{\Omega_{n} r^{n}} \int_{|t|<r}|f(x-t)| d t
$$

where $\Omega_{n}$ is the volume of the unit ball $\left\{t \in E_{n}:|t| \leqslant 1\right\}$. If we consider the space $M^{p}\left(E_{n}\right)=\left\{f \in L^{1}\left(E_{n}\right): m_{f} \in L^{p}\right\}, 1 \leqslant p<\infty$, and define $\|f\|$ for

Presented to the Society, June 16, 1978; received by the editors February 22, 1978.

AMS (MOS) subject classifications (1970). Primary 42A40, 42A96.

Key words and phrases. Segal algebras, homogeneous Banach spaces, Hardy-Littlewood maximal functions, Hilbert transforms, Sobolev spaces, nonfactorization.

'This research was conducted while the author was supported by National Science Council, Republic of China, on sabbatical leave from National Tsing Hua University, Republic of China, as a Visiting Fellow at Princeton University. 
$\operatorname{each} f \in M^{p}$ by

$$
\|f\|=\|f\|_{L^{\prime}}+\left\|m_{f}\right\|_{L^{p}},
$$

then \|\| is a norm on $M^{p}$, since $m_{f}$ is a sublinear operator and the $L^{p}$-norm is norm preserving. Let $\left(f_{n}\right)$ be a Cauchy sequence in $M^{p}$. Then $f_{n} \rightarrow f$ is the $L^{1}$-norm for some $f$ in $L^{1}\left(E_{n}\right)$. For $\varepsilon>0$ there exists a positive integer $N$ such that $\left\|m_{f_{n}-f_{m}}\right\|_{L^{p}}<\varepsilon$ whenever $n, m \geqslant N$. Without loss of generality we may assume $f_{n}$ converges to $f$ almost everywhere.

We have, for $m \geqslant N$,

$$
\begin{aligned}
\left\|m_{f-f_{m}}\right\|_{L^{p}}^{p} & =\int_{E_{n}}\left(m_{f-f_{m}}(x)\right)^{p} d x \\
& =\int_{E_{n}}\left[\sup _{r>0} \frac{1}{\Omega_{n} r^{n}} \int_{|t|<r}\left|\left(f-f_{m}\right)(x-t)\right| d t\right]^{p} d x \\
& \leqslant \underset{n \rightarrow \infty}{\lim } \int_{E_{n}}\left[\sup _{r>0} \frac{1}{\Omega_{n} r^{n}} \int_{|t|<r}\left|\left(f_{n}-f_{m}\right)(x-t)\right| d t\right]^{p} d x \\
& \leqslant \underset{n \rightarrow \infty}{\lim }\left\|m_{f_{n}-f_{m}}\right\|_{L^{p}}^{p} \leqslant \varepsilon^{p} .
\end{aligned}
$$

Therefore $f \in M^{p}$ and $\left\|f_{n}-f\right\| \rightarrow 0$ as $m \rightarrow \infty$. This asserts that $M^{p}$ is a Banach space. Recall that, for a finite ball $B \subset E_{n}$,

$$
\begin{aligned}
\left(m_{f_{x}}\right) & =\left(m_{f}\right)_{x}, \\
L^{1} \cap L^{p}\left(E_{n}\right) & \subset M^{p}\left(E_{n}\right), \quad 1<p<\infty, \\
C_{c}(B) & \subset M^{1}(B) \quad(\text { see Stein }[6, \text { p. 23] }) .
\end{aligned}
$$

We thus obtain:

1.1. TheOREM. $M^{p}\left(E_{n}\right), 1 \leqslant p<\infty$, and $M^{p}\left(T_{n}\right), 1 \leqslant p<\infty$, is a Segal algebra, where $T_{n}$ is the n-torus.

The maximal functions of Hardy-Littlewood control the estimates of Hilbert transforms and many others.

2. Hilbert transform. Given a function $f \in L^{1}\left(E_{1}\right)$, the Hilbert transform $\tilde{f}$ of $f$ is defined at $x$ by

$$
\tilde{f}(x)=\lim _{\varepsilon \rightarrow 0} \frac{1}{\pi} \int_{0<\varepsilon<|t|} \frac{f(x-t)}{t} d t .
$$

For $1<p<\infty$, let $H^{p}\left(E_{1}\right)$ be the space of all $f \in L^{1}\left(E_{1}\right)$ with $\tilde{f} \in L^{p}\left(E_{1}\right)$. Define

$$
\|f\|=\|f\|_{L^{1}}+\|\tilde{f}\|_{L^{p}} \quad\left(f \in H^{p}\right) .
$$

Since $\sim$ is linear, \|\| is a norm. Let $\left(f_{n}\right)$ be a Cauchy sequence in $H^{p}$. Take $f \in L^{1}\left(E_{1}\right), g \in L^{p}\left(E_{1}\right)$ such that $\left\|f_{n}-f\right\|_{L^{1}} \rightarrow 0$ and $\left\|\tilde{f}_{n}-g\right\|_{L^{p}} \rightarrow 0$. We have $\left\|\hat{f}_{n}-\hat{f}\right\|_{L^{\infty}} \rightarrow 0$ and $i \operatorname{sgn} x \hat{f}_{n} \rightarrow \hat{g}$ in the sense of distributions. Therefore $i \operatorname{sgn} x \hat{f}=\hat{g}$, or $\tilde{f}=g$. Therefore $H^{p}$ is complete. Recall that $\left(\tilde{f}_{x}\right)=$ 
$(\tilde{f})_{x}, L^{1} \cap L^{p}\left(E_{1}\right) \subset H^{p}$ for $1<p<\infty$, but if $f \in L^{1}\left(E_{1}\right), f>0$, then $\tilde{f} \notin L^{1}\left(E_{1}\right)$ (see Stein [6, p. 48]). We obtain:

2.1. THEOREM. $H^{p}\left(E_{1}\right), 1<p<\infty$, is a Segal algebra, but $H^{1}\left(E_{1}\right)$ is a non-Segal homogeneous Banach algebra.

The story of Hilbert transform on the circle group $T$ is somewhat different. Given a function $f \in L^{1}(T)$, the Hilbert transform $\tilde{f}$ of $f$ is defined at $x$ by

$$
\tilde{f}(x)=\lim _{\varepsilon \rightarrow 0} \frac{1}{2 \pi} \int_{\varepsilon<|t|<\pi} f(x-t) \cot \frac{t}{2} d t .
$$

In this case, if $f \log ^{+}|f| \in L^{1}(T)$, then $\tilde{f} \in L^{1}(T)$. We have

2.2. ThEOREM. $H^{p}(T), 1 \leqslant p<\infty$, is a Segal algebra.

2.3. Remarks. (i) For $f \in L^{p}\left(E_{1}\right), 1<p<\infty, \hat{\tilde{f}}(t)=-i \operatorname{sgn}(t) \hat{f}(t)$ in the sense of distributions. Therefore $-\tilde{\tilde{f}}=f$ or $H^{p}\left(E_{1}\right)=L^{1} \cap L^{p}\left(E_{1}\right)$ for $1<p$ $<\infty$. We conclude that $H^{p}\left(E_{1}\right), 1<p<\infty$, is character invariant.

(ii) Let $f \in H^{1}$ with $\hat{f}(-t) \neq 0$. Then $-i \operatorname{sgn}(x) \hat{f}(x-t)$ is discontinuous at 0 , or $e^{i t x} f(x) \notin H^{1}$. Therefore $H^{1}\left(E_{1}\right)$ is not character invariant.

One more important operator on Euclidean spaces comes to our attention:

3. Sobolov spaces. For $1 \leqslant p<\infty, k$ a positive integer, denote by $S_{k}^{p}\left(E_{n}\right)$ the space of all functions $f$ in $L^{1}\left(E_{n}\right)$ with $\partial^{\alpha} f / \partial x^{\alpha} \in L^{p}\left(E_{n}\right)$ for $0<|\alpha| \leqslant k$. For $f \in S_{k}^{p}$, define

$$
\|f\|=\|f\|_{L^{1}}+\sum_{0<|\alpha|<k}\left\|\frac{\partial^{\alpha} f}{\partial x^{\alpha}}\right\|_{L^{p}} .
$$

Then \|\| is a norm. This is because $T$ is linear where $T(f)=$ $\left(f, \ldots, \partial^{\alpha} f / \partial x^{\alpha}, \ldots\right) \in L^{1}\left(E_{n}\right) \times L^{p}\left(E_{n}\right) \times \cdots \times L^{p}\left(E_{n}\right)$. Now let $\left(f_{m}\right)$ be a Cauchy sequence in $S_{k}^{p}$. Take $f \in L^{1}\left(E_{n}\right), f^{(\alpha)} \in L^{p}\left(E_{n}\right)$ such that $\left\|f_{m}-f\right\|_{L^{1}} \rightarrow 0$, and $\left\|f_{m}^{(\alpha)}-f^{(\alpha)}\right\|_{L^{p}} \rightarrow 0$ where $f_{m}^{(\alpha)}=\partial^{\alpha} f_{m} / \partial x^{\alpha}$. For $\varphi \in$ $C_{c}^{\infty}\left(E_{n}\right)$, we have

$$
\begin{aligned}
\int_{E_{n}} f^{(\alpha)} \varphi & =\int_{E_{n}} \lim _{m}\left(f_{m}^{(\alpha)} \varphi\right) \\
& =\lim _{m} \int_{E_{n}} f_{m}^{(\alpha)} \varphi=\lim _{m}(-1)^{|\alpha|} \int_{E_{n}} f_{m} \varphi^{(\alpha)} \\
& =(-1)^{|\alpha|} \int_{E_{n}}\left(\lim _{m} f_{m}\right) \varphi^{(\alpha)}=(-1)^{|\alpha|} \int_{E_{n}} f \varphi^{(\alpha)}
\end{aligned}
$$

Hence $f^{(\alpha)}=\partial^{\alpha} f / \partial x^{\alpha}$ or $S_{k}^{p}$ is complete. It is easy to see that $T f_{x}=(T f)_{x}$ and that the space of testing functions is contained in $S_{k}^{p}$. We obtain:

3.1. TheOREM. $S_{k}^{p}\left(E_{n}\right)$ is a Segal algebra.

We call $S_{k}^{p}\left(E_{n}\right)$ the Sobolov algebra. Meanwhile two variant forms of Sobolov algebras are often considered: (I) For a positive integer $k$, the space 
$A_{k}^{P}\left(E_{n}\right)$ of all functions $f$ in $L^{1}\left(E_{n}\right)$ with $t^{k} \hat{f}(t) \in L^{p}\left(E_{n}\right)$ for $1 \leqslant p<\infty$, under the norm $\|f\|=\|f\|_{L^{1}}+\left\|t^{k} \hat{f}\right\|_{L^{p}}$. (II) For a real number $s$, the space $B^{s}\left(E_{n}\right)$ of all functions $f$ in $L^{1}\left(E_{n}\right)$ with $\left(1+|t|^{2}\right)^{s / 2} \hat{f}(t) \in L^{2}\left(E_{n}\right)$ under the norm $\|f\|=\|f\|_{L^{1}}+\left\|\left(1+|t|^{2}\right)^{s / 2} \hat{f}\right\|_{L^{2}}$.

There are closed relationships among the spaces $A_{k}^{p}, B^{s}$ and the Sobolov algebra $S^{p}$ just proved:

(i) $\left(\partial^{\alpha} f / \partial x^{\alpha}\right)^{\hat{n}}(t)=(2 \pi i t)^{\alpha} \hat{f}(t)$,

(ii) $|t|^{k}<\left(1+|t|^{2}\right)^{k / 2}$,

(iii) $c_{n}\left(1+|t|^{2}\right)^{-(n+1) / 2}$ is the Fourier transform of the Poisson kernel on $E_{n}$, where $c_{n}$ is a constant.

(iv) If we put $\left(\Lambda^{s} \mu\right)^{\wedge}(t)=\left(1+|t|^{2}\right)^{s / 2} \hat{\mu}(\xi)$, then $\Lambda^{s}=\left[1-(2 \pi)^{-2} \Delta\right]^{s / 2}$, where $\Delta$ is the Laplacian.

That the spaces $A_{k}^{p}$ and $B^{s}$ are Segal algebras is a consequence of Theorem 3.4.

3.2. Definition. A character function space is a Banach space $(A,\|\|)$ of measurable functions on $\Gamma$, in which two functions are the same if they are equal almost everywhere. Also the followings are satisfied

(i) If $\left\|f_{n}-f\right\|_{A} \rightarrow 0$, then there is a subsequence $\left(f_{n_{k}}\right)$ such that $f_{n_{k}}$ converges to $f$ almost everywhere.

(ii) If $f \in A, x \in G$, then $x f \in A$ and $\|x f\|_{A}=\|f\|_{A}$.

(iii) For $f \in A, \varepsilon>0$, there are $g, h \in A$ such that $f=g+h,\|h\|<\varepsilon$ and $x \rightarrow x g$ is a continuous map of $G$ into $A$.

3.3. ExAmpLes. (a) For a Segal algebra $\left(S(G),\|\|_{S}\right)$. Let $\|\hat{f}\|=\|f\|_{S}$, then $(\hat{S}(G),\|\|)$ is a character function space.

(b) Let $\mu$ be a regular unbounded measure on $\Gamma$, then $L^{p}(\mu)$ is a character function space.

(c) $C_{0}(\Gamma)$ is a character function space.

3.4. THEOREM. Let $S(G)$ be a Segal algebra, $A$ a character function space on $\Gamma$, and $\xi$ a function on $\Gamma$ with $\xi \hat{P}\left(L^{1}\right) \subset A$, where $P\left(L^{1}\right)$ is the space of all $f$ in $L^{1}(G)$ whose Fourier transforms $\hat{f}$ are compactly supported. Then the space $S(\xi$, $A)=\{f \in S(G): \xi \hat{f} \in A\}$ is a Segal algebra under the norm $\|f\|=\|f\|_{S}+$ $\|\xi f\|_{A}$.

Proof. Let $\left(f_{n}\right)$ be a Cauchy sequence in $S(\xi, A)$. Take $f \in S(G), g \in A$, such that $\left\|f_{n}-f\right\|_{s} \rightarrow 0$ and $\left\|\xi \hat{f}_{n}-g\right\|_{A} \rightarrow 0$. Thus $\xi \hat{f}=g$ so $S(\xi, A)$ is complete. For $f \in S(\xi, A), x \in G$, we have $f_{x} \in S(\xi, A)$ and $\left\|f_{x}\right\|=\|f\|$. We claim that $x \rightarrow f_{x}$ is continuous. For $\varepsilon>0$ there are $I, H \in A$ such that $\xi \hat{f}=I+H,\|H\|<\varepsilon / 8$, and $x \rightarrow x I$ is continuous. Choose a neighborhood $U$ of 0 in $G$ with $\|x I-I\|_{A}<\varepsilon / 4$ and $\left\|f_{x}-f\right\|_{S}<\varepsilon / 4$ whenever $x \in U$. We have $\left\|f_{x}-f\right\|<\varepsilon$ whenever $x \in U$. Therefore $S(\xi, A)$ is a homogeneous Banach space. Since $P\left(L^{1}\right) \subset S(\xi, A), S(\xi, A)$ is a Segal algebra.

The following Segal algebra given by Goldberg [2] and Riemersma [5] is a special case of Theorem 3.4. 
3.5. Corollary. Let $\alpha$ be a locally bounded function on $\Gamma$ with $\alpha \geqslant 1$. Denote $S(\alpha)$ by the space of all $f$ in $L^{1}(G)$ such that $\lim \hat{f}(\gamma) \alpha(\gamma)=0$. Under the norm $\|f\|_{\alpha}=\|f\|_{L^{1}}+\sup _{\gamma \in \Gamma}|\hat{f}(\alpha) \alpha(\gamma)|, S(\alpha)$ forms a Segal algebra.

In the Segal algebras $S(\xi, A)$, if we specialize the roles $S(G), \xi, A(\Gamma)$ such as Riemersma [5] and Wang [7, p. 81], we can answer every open question given by Reiter [3, p. 131].

4. In order to consider the nonfactorization property of these Segal algebras, we prove:

4.1. THEOREM. For $1 \leqslant p<\infty, 1<q<\infty, k$ a positive integer, the proper Segal algebras $M^{p}\left(E_{n}\right), H^{q}\left(E_{1}\right), S^{k}\left(E_{n}\right), S_{1}^{k}\left(E_{n}\right), S_{2}^{k}\left(E_{n}\right)$ are character invariant.

Proof. By definition, $M^{p}$ and $S^{k}$ are character invariant and by Remark 2.3, $H^{q}\left(E_{1}\right)=L^{1} \cap L^{q}\left(E_{1}\right)$. It suffices to prove that $S_{1}^{k}\left(E_{n}\right)$ and $S_{2}^{k}\left(E_{n}\right)$ are character invariant. But this follows from the inequalities:

$$
\begin{aligned}
|\xi+\eta|^{k} & \leqslant(|\xi|+|\eta|)^{k}, \\
\left(1+|\xi|^{2}\right)^{s / 2} & \leqslant 2^{|s|}\left(1+|\xi-\eta|^{2}\right)^{|s| / 2}\left(1+|\eta|^{2}\right)^{s / 2},
\end{aligned}
$$

where $k$ is a positive integer, $s \in E_{1}, \xi, \eta \in E_{n}$.

Feichtinger-Graham-Lakien [1] proved that a character invariant Segal algebra has factorization if and only if it is $L^{1}(G)$. As a consequence of their result, each Segal algebra in Theorem 4.1 does not have factorization. As a matter of fact, if $\xi(x+y) \leqslant \eta(x) \xi(y)$ for some function $\eta$, then the Segal algebra $S(\xi, A)$ has factorization if and only if $S(\xi, A)=L^{1}$. Note that $L^{1}(1$, $\left.C_{0}\right)=L^{1}$.

5. One of the most interesting features of Segal algebras is the richness of examples. We would like to point out here that the methods described can be used to investigate known Segal algebras.

As a result of Segal algebras, approximations to the identity in some important function spaces related to the operators mentioned can be easily derived.

ACKNOWLEDGEMENT. I wish to express my gratitude to Elias M. Stein for his lectures drawing my attention to the problem studied in this paper.

\section{REFERENCES}

1. H. G. Feichtinger, C. C. Graham and E. H. Lakien, Factorization in commutative weakly self-adjoint Banach algebras, Pacific J. Math. (to appear).

2. R. R. Goldberg, Recent results on Segal algebras, Lecture Notes in Math., vol. 399, Springer-Verlag, Berlin, 1974.

3. H. J. Reiter, Classical harmonic analysis and locally compact groups, Oxford Mathematical Monographs, Oxford Univ. Press, Oxford, 1968.

4. Berlin, 1971. 
5. M. Riemersma, On some properties of normed ideals on $L^{1}(G)$, Indag. Math. 37 (1975), 265-272.

6. E. M. Stein, Singular integrals and differentiability properties of functions, Princeton Univ. Press, Princeton, N. J., 1970.

7. H. C. Wang, Homogeneous Banach algebras, Lecture Notes in Pure and Appl. Mathematics, Dekker, New York, 1977.

Department of Mathematics, Princeton University, Princeton, New Jersey 08540

Department of Mathematics, National Tsing Hua Universtry, Tatwan, Republic of ChInA (Current address) 